\title{
Bell nonlocality using tensor networks and sparse recovery
}

\author{
I. S. Eliëns $\odot,{ }^{1}$ S. G. A. Brito $\odot,{ }^{1}$ and R. Chaves $\odot^{1,2}$ \\ ${ }^{1}$ International Institute of Physics, Federal University of Rio Grande do Norte, 59078-970 Natal, Brazil \\ ${ }^{2}$ School of Science and Technology, Federal University of Rio Grande do Norte, 59078-970 Natal, Brazil
}

(Received 3 February 2020; accepted 21 April 2020; published 20 May 2020)

\begin{abstract}
Bell's theorem, stating that quantum predictions are incompatible with a local hidden variable description, is a cornerstone of quantum theory and at the center of many quantum information processing protocols. Over the years, different perspectives on nonlocality have been put forward as well as different ways to detect nonlocality and quantify it. Unfortunately, and in spite of its relevance, as the complexity of the Bell scenario increases, deciding whether a given observed correlation is nonlocal becomes computationally intractable. Here, we propose to analyze a Bell scenario as a tensor network, a perspective permitting us to test and quantify nonlocality, resorting to very efficient algorithms originating from compressed sensing and that offer a significant speedup in comparison with standard linear programming methods. We use that all nonsignaling correlations can be described by hidden variable models governed by a quasiprobability, a fact we prove with simple linear algebra methods.
\end{abstract}

DOI: 10.1103/PhysRevResearch.2.023198

\section{INTRODUCTION}

Bell's theorem [1] shows that quantum predictions are at odds with the physical intuition from classical physics. More precisely, that the correlations obtained by local measurements on distant but entangled systems cannot be reproduced by any local hidden variable model, the phenomenon generally known as Bell nonlocality [2]. Historically a topic in the foundations of quantum theory, with the establishment of quantum information science, nonlocality is now understood as a resource in a number of information processing applications ranging from randomness certification [3], secure communication [4], reduction in communication complexity [5], and self-testing [6]. It is also at the core of the deviceindependent framework [7] where information processing is achieved without the need of a precise knowledge of the internal physical mechanisms of the state preparation and measurement apparatuses.

A central problem in the study of nonlocality is to decide whether a given observed correlation is nonlocal [2] and, furthermore, quantify it [8]. The standard approach is that based on Bell inequalities, experimentally testable witnesses, the violation of which allows for the device-independent certification of the nonlocal nature of the correlations under test. The set of correlations compatible with a local hidden variable model is characterized by a convex set [9], the nontrivial facets of which are precisely the Bell inequalities. However, the full characterization of Bell inequalities bounding a given scenario can only be achieved for the simplest cases [2] and

Published by the American Physical Society under the terms of the Creative Commons Attribution 4.0 International license. Further distribution of this work must maintain attribution to the author(s) and the published article's title, journal citation, and DOI. in practice one often has to rely on an incomplete set of inequalities [10-12]. Alternatively, the nonlocal behavior of a given correlation can be tested directly, resorting to linear programming (LP) $[2,13]$. Notwithstanding, the LP approach also suffers from the curse of dimensionality, being of no use as the number of parties, measurements, settings, or measurement outcomes increase in the Bell scenario.

Motivated by these issues [9,14-18], our aim in this paper is to offer an alternative view on Bell nonlocality, based on tensor networks [19] and sparse recovery [20-23]. We also drew inspiration from category theory and its applications to quantum mechanics and probability theory which in some sense represents a formal counterpart to the computationfocused tensor-network approach [24-29].

Tensor networks have become an important tool in condensed-matter physics, and constitute a field in rapid development that branches out into topics as varied as quantum gravity and machine learning [19]. The current surge of progress can be traced back to the invention of the density matrix renormalization group (DMRG) [30] and its reformulation in terms of matrix product states (MPSs) [31]. The successful application of tensor networks, as well as many machine learning models such as neural networks [32], rest on a combination of two factors: (i) The computational problem at hand allows for efficient encoding in terms of the given model and (ii) there is an efficient manner to fix the free parameters of the model by means of an optimization problem. In the case of finding the ground state of one-dimensional systems, the model is given by the MPS ansatz and DMRG is the optimization algorithm. In machine learning, one may think of neural networks and gradient descent.

Here we show that the problem of determining Bell nonlocality has a natural representation as a tensor network problem. It turns out that the optimization one has to perform is equivalent to the problem of basis pursuit known from the theory of compressed sensing. Compressed sensing refers to 
the idea that sparse signals can be reconstructed efficiently from a very limited number of observations (well below the Nyquist-Shannon limit) [20,21]. This allows one to recover a signal from a small number of observed data points by using convex optimization for recovery. This has lead to many applications in the last decade [22,23].

Based on the tensor network approach, we show a number of results. First, we show that nonsignaling correlations (including nonlocal correlations) can be mapped to hidden variable models governed by quasiprobabilities, that is, quantities that sum up to one but are not necessarily positive $[33,34]$. This points out a way to detect and quantify nonlocality with tools originating from the field of compressed sensing [35]. Nicely, the negativity of this quasiprobability provides a natural way to quantify nonlocality. Second, we provide a tensor network decomposition for the hidden variable model that introduces a natural basis to express the problem. We show that, formulated this way, sparse recovery algorithms allow for a significant speedup in the detection of nonlocality in comparison with the standard LP approach.

\section{BELL SCENARIO AS A TENSOR NETWORK}

We will focus here on the standard bipartite Bell scenario in which Alice and Bob each locally perform an experiment in spatially separated regions of space-time. However, all our results generalize in a straightforward manner to more parties. Alice and Bob have the freedom to choose experimental settings, labeled $x$ and $y$, respectively, and obtain outcomes indexed by labels $a$ and $b$ with some probability. The setup is fully described by a conditional probability $P(a b \mid x y)$, i.e., the probability to obtain outcome $a$ and $b$ given the inputs $x$ and $y$. We will call $\boldsymbol{P}$ a behavior or correlation.

To be consistent with the laws of special relativity, the behavior $\boldsymbol{P}$ must obey the nonsignaling conditions:

$$
\begin{aligned}
& \sum_{b} P(a b \mid x y)=\sum_{b} P\left(a b \mid x y^{\prime}\right) \text { for all } a, x, y, y^{\prime}, \\
& \sum_{a} P(a b \mid x y)=\sum_{a} P\left(a b \mid x^{\prime} y\right) \text { for all } b, y, x, x^{\prime} .
\end{aligned}
$$

In a quantum description, according to Born's rule, the probability distribution in a Bell scenario should be given by

$$
P(a b \mid x y)=\operatorname{Tr}\left[\left(M_{a}^{x} \otimes M_{b}^{y}\right) \rho\right],
$$

where $\rho$ is the density matrix describing the quantum state shared between Alice and Bob and $M_{a}^{x}$ and $M_{b}^{y}$ are positive-operator valued measure (POVM) operators describing their measurements. Clearly, quantum correlations are nonsignaling, however, there are nonsignaling correlations of a postquantum nature [36].

Moreover, if the experimental outcomes can be explained within the assumptions of local realism, the conditional probability allows a decomposition:

$$
P(a b \mid x y)=\sum_{\lambda} P(a \mid x \lambda) P(b \mid y \lambda) p(\lambda) .
$$

It is well known that we can replace the local conditional probabilities [e.g., $P(a \mid x \lambda)$ for Alice] by a deterministic process mapping each $x$ to some $a$ (i.e., a function) and the local variable simply determines the probability for the combination of deterministic processes at Alice's and Bob's side. This means, as shown by Fine [37], that a local hidden variable (LHV) model can equivalently be defined as a joint probability distribution $p\left(a_{1}, \ldots, a_{m}, b_{1}, \ldots, b_{m}\right)$. Hence, a set of marginals $P(a b \mid x y)$ is called local if it is consistent with the existence of this joint probability distribution for all measurements. In other words, $\lambda$ can be taken to be the combination of two sequences $\left(a_{1} \ldots a_{m}\right)$ and $\left(b_{1} \ldots b_{m}\right)$ that prescribe the outputs $a_{x}$ and $b_{y}$ for each of the inputs $x$ and $y$, and we can write

$$
P(a b \mid x y)=\sum_{a_{1} \ldots a_{m}} \sum_{b_{1} \ldots b_{m}} \delta_{a a_{x}} \delta_{b b_{x}} q_{a_{1} \ldots a_{m} b_{1} \ldots b_{m}},
$$

where $q_{a_{1} \ldots a_{m} b_{1} \ldots b_{m}}$ is the corresponding probability $\boldsymbol{q}=\boldsymbol{p}$. Remarkably, as we will show, any nonsignaling conditional probability allows such a decomposition with $\boldsymbol{q}$ a quasiprobability, i.e., $q_{a_{1} \ldots a_{m} b_{1} \ldots b_{m}}$ can take negative values but still sums to 1 . This was noted in Ref. [14] but in slightly different form and totally different language. We will arrive at this observation independently from a reasoning rooted in tensor network theory. Furthermore, the decomposition in Eq. (4) gives another approach for testing Bell nonlocality: One can now search the space of all $\boldsymbol{q}$ compatible with $\boldsymbol{P}$ for an element with only non-negative coefficients, i.e., a proper probability (see Sec. IV).

As we will see, the appearance of the deterministic matrix $\boldsymbol{D}$ with coefficients $D_{a x, a_{1} \ldots a_{m}}=\delta_{a a_{x}}$ in Eq. (4) naturally follows from the nonsignaling condition (a consequence of Lemma 1 in Sec. III). The matrix $\boldsymbol{D}$ turns out to have an interesting decomposition. The second aim of our paper is to formalize this structure related to the decomposition of $\boldsymbol{D}$, present in the Bell nonlocality problem. We find that tensor networks provide a natural language for this, in particular, with generalization to multipartite scenarios and different causal structures in mind.

Let us establish some conventions and notations. Any object with multiple indices such as a conditional probability $P(a b \mid x y)$ or a (quasi) probability $q_{a_{1} \ldots a_{m}}$ will be viewed as a tensor [38]. We will use mixed notations for indices-upper, lower, as function argument-without distinction. We will assume that $x$ and $y$ take values in $1, \ldots, m$ while $a, b$ take values in $0, \ldots, n-1$, unless stated otherwise. If any or all of the indices are suppressed, we will use bold face such as $\boldsymbol{P}$ and $\boldsymbol{q}$. The graphical depiction of a tensor as a box with a line for each index is often useful. For example, $\boldsymbol{P}$ we depict as

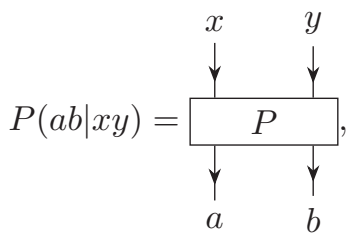

where we used an arrow on the lines to distinguish input from output indices. Connecting lines between tensors implies summation over the corresponding index, also called contraction. Any number of tensors with specified contractions constitutes a tensor network. A scalar $c$, a vector $v_{a}$, and a matrix $M_{a b}$ are tensors with, respectively, 0,1 and 
2 indices. It is often useful to group indices of a tensor into a multi-index. This way, any tensor $T_{a_{1} \ldots a_{m}}$ can be viewed as a single vector with $n^{m}$ elements with indices $\left(a_{1} \ldots a_{m-1} a_{m}\right)=(0 \ldots 00),(0, \ldots 01) \ldots(1 \ldots 11)$. We use row-major/lexicographic order. By splitting the indices in two groups, we can view $\boldsymbol{T}$ as a matrix $T_{a_{1} \ldots a_{r}, a_{r+1} \ldots a_{m}}$. We often use the same letter with different indices to denote different tensors.

With the above definitions in place, the question whether $\boldsymbol{P}$ is local Eq. (3) or quantum Eq. (2) is trivially a question whether or not $\boldsymbol{P}$ admits a certain type of tensor network decomposition. Realizing this does, however, not make the question any easier to answer per se. In the remainder of this paper, we discuss how realizing that nonsignaling correlations can also be defined in terms of such a tensor network decomposition as in Eq. (4) and applying a further decomposing of $\boldsymbol{D}$ can be useful in determining the nonlocality of a given $\boldsymbol{P}$.

\section{THE DECOMPOSITION OF NONSIGNALING CORRELATIONS}

In this section, we discuss the tensor-network decomposition of nonsignaling correlations. First, we prove Eq. (4) for any nonsignaling $P(a b \mid x y)$. Then we discuss the singular value decomposition (SVD) of the deterministic tensor $\boldsymbol{D}$ with coefficients $\delta_{a a_{x}}$, which results in a full tensor network decomposition of $\boldsymbol{P}$. The decomposition is depicted in Fig. 1. We furthermore discuss how the transformation to a certain choice of basis of singular vectors of $\boldsymbol{D}$ is a natural generalization of the correlation representation of a probability.

Recall that any matrix $\boldsymbol{M}$ allows a SVD $\boldsymbol{M}=\boldsymbol{U} \boldsymbol{S} \boldsymbol{V}^{\dagger}$ where $\boldsymbol{U}, \boldsymbol{V}$ are unitary (orthogonal if $\boldsymbol{M}$ is real) and $\boldsymbol{S}$ is quasidiagonal.

Lemma 1. A matrix $M_{a x}$ has constant column sums $\sum_{a} M_{a x}=\mathcal{C}$ (independent of $x$ ) iff it can be decomposed as $M_{a x}=\sum_{a_{1} \ldots a_{m}} \delta_{a a_{x}} C_{a_{1} \ldots a_{m}}$ with $\sum_{a_{1} \ldots a_{m}} C_{a_{1} \ldots a_{m}}=\mathcal{C}$. If all $M_{a x} \geqslant 0$, then we can choose all $C_{a_{1} \ldots a_{m}} \geqslant 0$. (Here we assume that $x$ takes values $1, \ldots, m$.)

Proof. To show the if statement, suppose that $M_{a x}=\sum_{a_{1} \ldots a_{m}} \delta_{a a_{x}} C_{a_{1} \ldots a_{m}}$. Summing over $a$ gives $\sum_{a} M_{a x}=$ $\sum_{a_{1} \ldots a_{m}} C_{a_{1} \ldots a_{m}}=\mathcal{C}$. Clearly, $M_{a x} \geqslant 0$ if $C_{a_{1} \ldots a_{m}} \geqslant 0$ and the statement follows.

To show the only if statement, let us start with the case $M_{a x} \geqslant 0$. We do induction on the column sum. For $\mathcal{C}=0$, the only option is $M_{a x}=0$ for all $a, x$. Suppose we proved the statement for $\mathcal{C}^{\prime} \leqslant \mathcal{C}$ and we are given $M_{a x} \geqslant 0$ with column sums $\mathcal{C}$. Pick the coefficients $a_{x}$ of the smallest nonzero elements of each column $x$ of $\boldsymbol{M}$ and let $\lambda$ be the smallest value of all these elements. Then matrix $\boldsymbol{M}^{\prime}$ with coefficients $M_{a x}^{\prime}=M_{a x}-\lambda \delta_{a a_{x}}$ and $M_{a^{\prime} x^{\prime}}^{\prime}=M_{a^{\prime} x^{\prime}}$ for $(a, x) \neq\left(a^{\prime}, x^{\prime}\right)$ has all coefficients non-negative and column sums $\mathcal{C}^{\prime}=\mathcal{C}-$ $\lambda \leqslant \mathcal{C}$. Hence, by the induction step, we can write $M_{a x}^{\prime}=$ $\sum_{a_{1}^{\prime} \ldots a_{m}^{\prime}} \delta_{a a_{x}^{\prime}} C_{a_{1}^{\prime} \ldots a_{m}^{\prime}}^{\prime}$ with $C_{a_{1} \ldots a_{m}}^{\prime} \geqslant 0$. Adding back $\lambda \delta_{a a_{x}}$ gives the required decomposition for $\boldsymbol{M}$. This establishes the lemma for this case.

Next, note that the matrices with a single 1 and a single -1 in one of the columns and otherwise zeros can be constructed as $N_{a x}=\delta_{a a_{x}}-\delta_{a a_{x}^{\prime}}$ where $\boldsymbol{a}=\left(a_{1}, \ldots, a_{m}\right)$ and $\boldsymbol{a}^{\prime}=\left(a_{1}^{\prime} \ldots, a_{m}^{\prime}\right)$ differ only for the label $x$ corresponding to

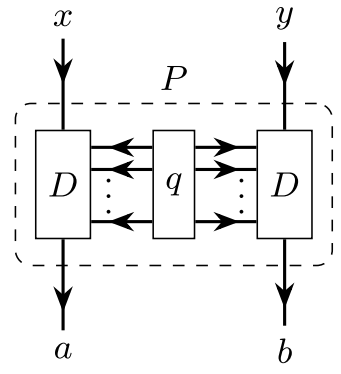

(a)

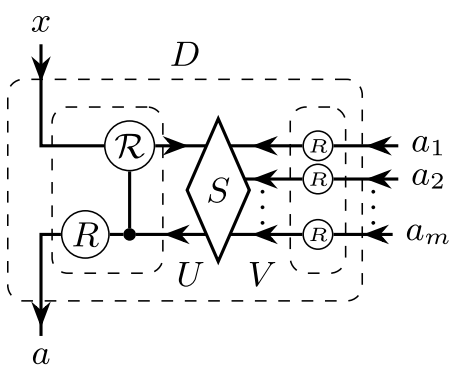

(b)

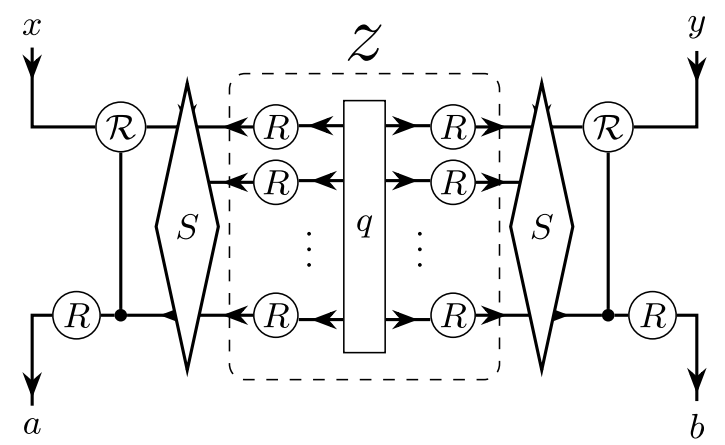

(c)

FIG. 1. Tensor-network decomposition of bipartite nonsignaling conditional probability $P(a b \mid x y)$. (a) The nonsignaling condition on $\boldsymbol{P}$ is equivalent to the decomposition $\boldsymbol{P}=(\boldsymbol{D} \otimes \boldsymbol{D}) \boldsymbol{q}$ with $\boldsymbol{q}$ a quasiprobability and $\boldsymbol{D}$ the deterministic tensor. (b) The singular value decomposition (SVD) of the deterministic tensor $\boldsymbol{D}$ has a nice tensor network representation. Writing $\boldsymbol{D}=\boldsymbol{U} \boldsymbol{S} \boldsymbol{V}^{T}$, we find that $\boldsymbol{U}$ and $\boldsymbol{V}$ are expressed in terms of the rotation $\boldsymbol{R}$. (c) We write the full decomposition of $\boldsymbol{P}$ as a tensor network. Indicated is the vector $\boldsymbol{z}=\boldsymbol{R}^{\otimes 2 m} \boldsymbol{q}$, i.e., the generalized correlation representation of the hidden-variable quasiprobability $\boldsymbol{q}$.

the column with $a_{x} \neq a_{x}^{\prime}$ the corresponding row indices of the nonzero coefficients. We can convert any matrix with constant column sums into any other matrix with the same constant column sums by adding a superposition of such matrices $N$. Combined with the case $M_{a x} \geqslant 0$, we have shown that the matrices $\delta_{a a_{x}}$ form an overcomplete set that generates all matrices with constant column sum and the lemma follows.

A conditional probability $P(a \mid x)$ can be viewed as a stochastic matrix with constant column sums 1 and nonnegative coefficients. The lemma states that this can be decomposed as a superposition of matrices with a single 1 in each column with non-negative coefficients, as in the following simple example:

$$
\left[\begin{array}{ll}
1 / 8 & 3 / 8 \\
7 / 8 & 5 / 8
\end{array}\right]=\frac{1}{8}\left[\begin{array}{ll}
1 & 0 \\
0 & 1
\end{array}\right]+\frac{3}{8}\left[\begin{array}{ll}
0 & 1 \\
1 & 0
\end{array}\right]+\frac{4}{8}\left[\begin{array}{ll}
0 & 0 \\
1 & 1
\end{array}\right]
$$

Such a decomposition is not necessarily unique. The white noise probability $P(a \mid x)=1 / n$ can, for example, easily be seen to allow different decompositions. 
To illustrate how the lemma can be used, let us obtain Eq. (4) from Eq. (3) in a single line of algebra:

$$
\begin{aligned}
P(a b \mid x y)= & \sum_{\lambda} P(a \mid x \lambda) P(b \mid y \lambda) p(\lambda) \\
= & \sum_{a_{1} \ldots a_{m}} \sum_{b_{1} \ldots b_{m}} \delta_{a a_{x}} \delta_{b b_{y}} \\
& \times \underbrace{\sum_{\lambda} P\left(a_{1} \ldots a_{m} \mid \lambda\right) P\left(b_{1} \ldots b_{m} \mid \lambda\right) p(\lambda)}_{q_{a_{1} \ldots a_{m} b_{1} \ldots b_{m}}} .
\end{aligned}
$$

Clearly, $q_{a_{1} \ldots a_{m} b_{1} \ldots b_{m}}$ is a probability, so we recover Fine's theorem. Here Lemma 1 was applied on the conditional probabilities $P(a \mid x \lambda)$ and $P(b \mid y \lambda)$ for fixed $\lambda$.

The non-negative case of the lemma states that stochastic matrices form a polytope with corners given by the matrices with coefficients $\delta_{a a_{x}}$, which are encoding functions or deterministic processes. As such, it can be viewed as a generalization of the Birkhoff-von Neumann theorem that states that doubly stochastic matrices form a polytope with corners given by the permutation matrices. Lemma 1 applied to double stochastic matrices is easily seen to imply this as a corollary.

Let $Q(a \mid x)$ define a quasistochastic matrix with column sums equal to 1 but some negative elements. In this case, we will always find some negative expansion coefficients in the superposition, such as in the following simple example:

$$
\left[\begin{array}{cc}
-1 / 8 & 3 / 8 \\
9 / 8 & 5 / 8
\end{array}\right]=-\frac{1}{8}\left[\begin{array}{ll}
1 & 1 \\
0 & 0
\end{array}\right]+\frac{4}{8}\left[\begin{array}{ll}
0 & 1 \\
1 & 0
\end{array}\right]+\frac{5}{8}\left[\begin{array}{ll}
0 & 0 \\
1 & 1
\end{array}\right] .
$$

Lemma 1 turns out to be quite versatile.

Let us now consider the bipartite $P(a b \mid x y)$ that satisfies the nonsignaling property and see how to derive the decomposition in Eq. (4) in terms of a quasiprobability hidden variable model. We can interpret $\boldsymbol{P}$ as a matrix by grouping Alice's and Bob's indices and write the SVD as $P(a b \mid x y)=$ $\sum_{\lambda} A_{a x}^{\lambda} \Lambda_{\lambda} B_{b y}^{\lambda}$. From the nonsignaling property, it is clear that $\sum_{a} A_{a x}^{\lambda}$ is independent of $x$ and $\sum_{b} B_{b y}^{\lambda}$ is independent of $y$ for fixed $\lambda$. Then, applying Lemma 1, we find that $A_{a x}^{\lambda}=\sum_{a_{1} \ldots a_{m}} \delta_{a a_{x}} \mathcal{A}_{a_{1} \ldots a_{m}}^{\lambda}$ and $B_{a x}^{\lambda}=\sum_{a_{1} \ldots a_{m}} \delta_{b b_{x}} \mathcal{B}_{b_{1} \ldots b_{m}}^{\lambda}$ for some $\mathcal{A}^{\lambda}$ and $\mathcal{B}^{\lambda}$. Hence we have found a decomposition of the form in Eq. (4) with $q_{a_{1} \ldots a_{m} b_{1} \ldots b_{m}}=\sum_{\lambda} \mathcal{A}_{a_{1} \ldots a_{m}}^{\lambda} \mathcal{B}_{b_{1} \ldots b_{m}}^{\lambda} \Lambda_{\lambda}$, which is real and summing over all indices gives 1 . However, in general, the coefficients of $\boldsymbol{q}$ can be negative, hence it is a quasiprobability.

To give a concrete example, let us put $n=m=2$ and consider the Popescu-Rohrlich (PR) box [36]:

$$
P(a b \mid x y)=\frac{1}{2} \delta_{a \oplus b, x y}
$$

(we use $a, b, x, y \in\{0,1\}$ and $a \oplus b=a+b \bmod 2$ ). We can find

$$
q_{a_{0} a_{1} b_{0} b_{1}}=\frac{1}{16}\left[\begin{array}{cccc}
3 & 3 & -1 & -1 \\
3 & -1 & 3 & -1 \\
-1 & 3 & -1 & 3 \\
-1 & -1 & 3 & 3
\end{array}\right]_{a_{0} a_{1}, b_{0} b_{1}}
$$

Doing the summation, we recover $P(a b \mid x y)=$ $\sum_{a_{0} a_{1} b_{0} b_{1}} \delta_{a a_{x}} \delta_{b b_{y}} q_{a_{0} a_{1} b_{0} b_{1}}$.

We can write Eq. (4) as a linear equation $\boldsymbol{P}=(\boldsymbol{D} \otimes \boldsymbol{D}) \boldsymbol{q}$. Here $\boldsymbol{D}$ is a tensor defined as the coefficients $D_{a x a_{1} \ldots a_{m}}=\delta_{a a_{x}}$ reshaped as a matrix. In Fig. 1(a), we draw this equation as a tensor network. Our next step is to refine this decomposition by a further decomposition of $\boldsymbol{D}$.

Let us take a look at the simplest case of two inputs and two outputs $a, b, x, y \in\{0,1\}$ for illustration. The deterministic matrix, in this case,

$$
\boldsymbol{D}=\left[\begin{array}{llll}
1 & 1 & 0 & 0 \\
1 & 0 & 1 & 0 \\
0 & 0 & 1 & 1 \\
0 & 1 & 0 & 1
\end{array}\right]
$$

can be seen to allow the decomposition $\boldsymbol{D}=\frac{1}{2} \boldsymbol{E} \boldsymbol{F}$ with

$\boldsymbol{E}=\left[\begin{array}{cccc}1 & 0 & 1 & 0 \\ 1 & 1 & 0 & 0 \\ 1 & 0 & -1 & 0 \\ 1 & -1 & 0 & 0\end{array}\right], \quad \boldsymbol{F}=\left[\begin{array}{cccc}1 & 1 & 1 & 1 \\ 1 & -1 & 1 & -1 \\ 1 & 1 & -1 & -1 \\ 1 & -1 & -1 & 1\end{array}\right]$

Let $q_{a_{0} a_{1}}$ be a joint probability for the two experiments $x=0,1$ of Alice and let $P(a \mid x)$ be the corresponding marginals for a single experiment. Writing $\boldsymbol{q}$ and $\boldsymbol{P}$ as two four-dimensional vectors, we find that

$$
\boldsymbol{F} \boldsymbol{q}=\left[\begin{array}{c}
1 \\
\left\langle a_{1}\right\rangle \\
\left\langle a_{0}\right\rangle \\
\left\langle a_{0} a_{1}\right\rangle
\end{array}\right], \quad \boldsymbol{E}^{T} \boldsymbol{P}=\left[\begin{array}{c}
1 \\
\left\langle a_{1}\right\rangle \\
\left\langle a_{0}\right\rangle \\
0
\end{array}\right]
$$

where we used $\left\langle a_{x}\right\rangle=\sum_{a}(-1)^{a} P(a \mid x)=\sum_{a_{0} a_{1}}(-1)^{a_{x}} q_{a_{0} a_{1}}$ and $\left\langle a_{0} a_{1}\right\rangle=\sum_{a_{0} a_{1}}(-1)^{a_{0}+a_{1}} q_{a_{0} a_{1}}$, the usual correlation representation for binary probabilities. Curiously, the two vectors in Eqs. (13) agree except for the nonobservable $\left\langle a_{0} a_{1}\right\rangle$. Since $\boldsymbol{E}^{T} \boldsymbol{E}=\operatorname{diag}(4,2,2,0)$, its value plays no role in the equality $\boldsymbol{P}=\boldsymbol{D} \boldsymbol{q}=\frac{1}{2} \boldsymbol{M} \boldsymbol{V} \boldsymbol{q}$ anyway.

The observations above are simply a manifestation of the SVD of $\boldsymbol{D}$, which we can find explicitly. We write

$$
\begin{aligned}
D_{a x a_{1} \ldots a_{m}} & =\delta_{a a_{x}} \\
& =\sum_{a^{\prime} x^{\prime}} \sum_{a_{1}^{\prime} \ldots a_{m}^{\prime}} U_{a x a^{\prime} x^{\prime}} S_{a^{\prime} x^{\prime} a_{1}^{\prime} \ldots a_{m}^{\prime}} V_{a_{1} \ldots a_{m} a_{1}^{\prime} \ldots a_{m}^{\prime} .}
\end{aligned}
$$

By a direct computation, one can check that the following definitions are consistent with this decomposition:

$$
\begin{aligned}
U_{a x a^{\prime} x^{\prime}} & =R_{a a^{\prime}} \mathcal{R}_{x x^{\prime}}^{a^{\prime}}, \\
S_{a x a_{1} \ldots a_{m}} & =\sqrt{n^{m-1}}\left[\sqrt{m} \delta_{0 a} \delta_{1 x}+1-\delta_{0 a}\right] \delta_{a a_{x}} \prod_{y \neq x} \delta_{0 a_{y}},
\end{aligned}
$$

$$
V_{a_{1} \ldots a_{m}, a_{1}^{\prime} \ldots a_{m}^{\prime}}=R_{a_{1} a_{1}^{\prime}} \ldots R_{a_{m} a_{m}^{\prime}} .
$$

The matrix $\boldsymbol{R}$ defines a rotation to a basis of which the first vector labeled by $b=0$ has all coefficients equal, while all 
other basis vectors $b>0$ have column sum zero,

$$
R_{a b}= \begin{cases}1 / \sqrt{n} & \text { for } b=0 \\ 1 / \sqrt{b(b+1)} & \text { for } a<b \neq 0 \\ -b / \sqrt{b(b+1)} & \text { for } a=b \neq 0 \\ 0 & \text { for } a>b \neq 0 .\end{cases}
$$

The tensor $\mathcal{R}$ is like the controlled version of $\boldsymbol{R}$,

$$
\mathcal{R}_{x y}^{a}=\delta_{0 a} R_{x y}+\left(1-\delta_{0 a}\right) \delta_{x y},
$$

which only implements the rotation when the control equals $a=0$ and which for $a \neq 0$ just acts as the identity, similar in spirit to the well-known Controled Not (CNOT) gate. Of course, in the definition of $R_{x y}$, we have to replace $n$ by $m$ when compared to Eq. (18). The tensor network representation of the SVD of $\boldsymbol{D}$ is depicted in Fig. 1(b).

Let us make a general definition: For a general (quasi) probability $q_{c_{1} \ldots c_{l}}$, we can use the rotation $\boldsymbol{R}$ to define

$$
z_{c_{1}^{\prime} \ldots c_{l}^{\prime}}=\sum_{c_{1} \ldots c_{l}} R_{c_{1} c_{1}^{\prime}} \ldots R_{c_{l} c_{l}^{\prime}} q_{c_{1} \ldots c_{l}}
$$

and we refer to the standard basis in the $c_{z}^{\prime}$ indices as the correlation basis. The reason is that transforming $\boldsymbol{q}$ to $z$, i.e., expressing $\boldsymbol{q}$ in the correlation basis, is a natural generalization of the correlation representation of binary probabilities to $n>2$.

Take the case of two measurement outcomes $n=2$. The matrix $\boldsymbol{R}$ is simply the Hadamard gate:

$$
\boldsymbol{R}=\frac{1}{\sqrt{2}}\left[\begin{array}{cc}
1 & 1 \\
1 & -1
\end{array}\right]
$$

This means that for a binary probability $q_{a}$ with $a=0,1$, the vector $z$ has only a single nontrivial coefficient,

$$
z=\boldsymbol{R}^{T} \boldsymbol{q}=\frac{1}{\sqrt{2}}\left[\begin{array}{c}
1 \\
\langle a\rangle
\end{array}\right],
$$

where $\langle a\rangle=\sum_{a}(-1)^{a} q_{a}$ is the usual correlation. For a probability $q_{a_{1} \ldots a_{m}}$ with all $a_{x} \in\{0,1\}$, we get $z=\left[\boldsymbol{R}^{\otimes m}\right]^{T} \boldsymbol{q}$ with coefficients

$$
\begin{aligned}
z_{0 \ldots 0} & =1 / \sqrt{2^{m}}, \\
z_{00 a_{x} \ldots 0} & =\left\langle a_{x}\right\rangle / \sqrt{2^{m}}, \\
z_{0 \ldots a_{x} \ldots 00 \ldots a_{y} \ldots 0} & =\left\langle a_{x} a_{y}\right\rangle / \sqrt{2^{m}}, \\
& \vdots
\end{aligned}
$$

The first element just encodes normalization of $\boldsymbol{q}$ while the other elements of $\boldsymbol{z}$ contain the correlations of $\boldsymbol{q}$ and all its marginals.

For $n=3$, one has

$$
\boldsymbol{R}=\left[\begin{array}{ccc}
1 / \sqrt{3} & 1 / \sqrt{2} & 1 / \sqrt{6} \\
1 / \sqrt{3} & -1 / \sqrt{2} & 1 / \sqrt{6} \\
1 / \sqrt{3} & 0 & -2 / \sqrt{6}
\end{array}\right],
$$

and we find

$$
z=\boldsymbol{R}^{T} \boldsymbol{q}=\left[\begin{array}{c}
1 / \sqrt{3} \\
\left(p_{0}-p_{1}\right) / \sqrt{2} \\
\left(p_{0}+p_{1}-2 p_{2}\right) / \sqrt{6}
\end{array}\right] .
$$

Since $\boldsymbol{R}$ is orthogonal, it is obvious that $\boldsymbol{q}$ and $z$ contain the same information, just expressed in different bases. Whereas, for $n=2$, the definition of correlation is unique up to normalization; for $n>2$, there is a freedom: We may take any basis in the subspace of vectors which sum to zero to define the columns of $\boldsymbol{R}$ (except the first). Conscious of this ambiguity in the case $n>2$ and despite the fact that this terminology is not standard, we will call the $z$ the (generalized) correlation representation of $\boldsymbol{q}$ and refer to its coefficients as (generalized) correlations. Rotating to the correlation basis may be useful in studying more general contextuality scenarios [39]. Note that we can recognize the matrices from Eqs. (12) as $\frac{1}{2} \boldsymbol{E}=\boldsymbol{U S}$ and $\frac{1}{2} \boldsymbol{F}=\boldsymbol{V}=\boldsymbol{R} \otimes \boldsymbol{R}$.

Going back to the bipartite behavior $P(a b \mid x y)$, we can now draw the full tensor-network decomposition of $\boldsymbol{P}$ as we do in Fig. 1(c). The vector $\boldsymbol{z}$ corresponding to $q_{a_{1} \ldots a_{m} b_{1} \ldots b_{m}}$ has coefficients

$$
\begin{aligned}
z_{0 \ldots 00 \ldots 0} & =n^{-m}, \\
z_{0 \ldots a_{x}^{\prime} \ldots 00 \ldots 0} & =n^{\frac{1}{2}-m} \sum_{a} R_{a a_{x}^{\prime}} P(a \mid x), \\
z_{0 \ldots 00 \ldots b_{y}^{\prime} \ldots 0} & =n^{\frac{1}{2}-m} \sum_{b} R_{b b_{y}^{\prime}} P(b \mid y), \\
z_{0 \ldots a_{x}^{\prime} \ldots 00 \ldots b_{y}^{\prime} \ldots 0} & =n^{1-m} \sum_{b} R_{a a_{x}^{\prime}} R_{b b_{y}^{\prime}} P(a b \mid x y),
\end{aligned}
$$

fixed by $P(a b \mid x y)$. All other $z_{a_{1} \ldots a_{m} b_{1} \ldots b_{m}}$ are free parameters as far as $P(a b \mid x y)$ is concerned, corresponding to the zero singular values of $\boldsymbol{D} \otimes \boldsymbol{D}$ defining its kernel.

In the optimization algorithm discussed in the next section, we search for $\boldsymbol{z}$ such that $\boldsymbol{q}$ has minimal negativity keeping the coefficients in Eqs. (26)-(29) fixed. If $\boldsymbol{z}$ exists such that $\boldsymbol{q}$ is non-negative, then $\boldsymbol{q}$ is a joint probability and according to Fine's theorem this means that $\boldsymbol{P}$ is local.

Lemma 1 and the explicit SVD of $\boldsymbol{D}$ [Eqs. (14)-(19)] represent the main technical results of this paper. The first implies the decomposition in Eq. (4) which, although known, we provide with a new derivation and utility. Refining the decomposition using the SVD of $\boldsymbol{D}$ gives the decomposition of $\boldsymbol{P}$ as an interesting tensor network and suggests to attack the problem of Bell nonlocality in a certain basis of singular vectors of $\boldsymbol{D} \otimes \boldsymbol{D}$ that we call the correlation basis.

\section{RECOVERING THE HIDDEN VARIABLE AND QUANTIFYING NONLOCALITY}

In this section, we turn to the question of determining and quantifying Bell nonlocality of a given nonsignaling $P(a b \mid x y)$. We will first discuss Bell inequalities for completeness. Then we turn to methods based on compressed sensing.

Bell inequalities are the traditional way to test Bell nonlocality, and the violation can be used to quantify nonlocality. The canonical example is the Clauser-Horne-Shimony-Holt (CHSH) inequality for the $n=m=2$ case, which written in terms of correlations reads

$$
\left|\left\langle a_{0} b_{0}\right\rangle+\left\langle a_{0} b_{1}\right\rangle+\left\langle a_{1} b_{0}\right\rangle-\left\langle a_{1} b_{1}\right\rangle\right| \leqslant 2 .
$$

With the last section in mind, we can of course write this in terms of the correlation basis $z_{a_{0} a_{1} b_{0} b_{1}}=$ 


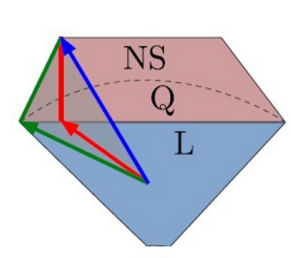

(a)

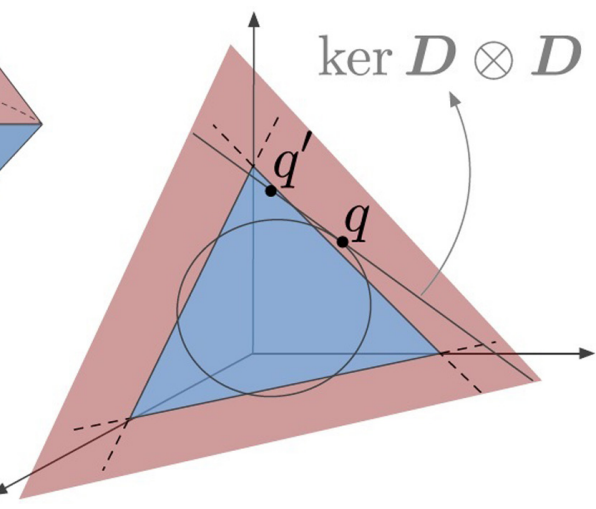

(b)

FIG. 2. Conceptual illustration of the local versus nonlocal correlations. (a) In the space of conditional probabilities $\boldsymbol{P}$, the nonsignaling condition defines a polytope (NS). Strictly included in NS there is the polytope of local correlations (L). We show that NS exactly corresponds to those $\boldsymbol{P}$ which allow a hidden variable model defined by a quasiprobability $\boldsymbol{q}$, i.e., solutions to the equation $(\boldsymbol{D} \otimes \boldsymbol{D}) \boldsymbol{q}=\boldsymbol{P}$. The local polytope L exactly corresponds to those $\boldsymbol{P}$ for which $\boldsymbol{q}$ can be chosen to be a probability. We have also illustrated a triangle in which we have sampled random behaviors for our comparison between NESTA and LP in Fig. 3. The blue, red, and green paths correspond to the plots of negativity and computation times in Fig. 4. The quantum set $\mathrm{Q}$ denotes a convex set with strict inclusions $\mathrm{L} \subset$ $\mathrm{Q} \subset \mathrm{NS}$ of $\boldsymbol{P}$ that can be obtained as a quantum mechanical expectation value. (b) The hyperplane affine plane of quasiprobabilities $\boldsymbol{q}$ (red) with the probability simplex indicated (blue). Probabilities $\boldsymbol{q}$ give local $\boldsymbol{P}$ via the equation $\boldsymbol{P}=(\boldsymbol{D} \otimes \boldsymbol{D}) \boldsymbol{q}$, but nonprobability solutions exist even when $\boldsymbol{P}$ is local. All solutions are related as $\boldsymbol{q}^{\prime}=\boldsymbol{q}+\boldsymbol{k}$ if $\boldsymbol{k} \in \operatorname{ker} \boldsymbol{D} \otimes \boldsymbol{D}$. To ensure that we find a probability $\boldsymbol{q}$ when $\boldsymbol{P}$ is local, we search for the solution with minimal $\ell_{1}$ norm.

$$
\begin{aligned}
& \sum(-1)^{a_{0}+a_{1}+b_{0}+b_{1}} q_{a_{0} a_{1} b_{0} b_{1}} / 4 \text { as } \\
& \qquad\left|z_{1010}+z_{0110}+z_{1001}-z_{0101}\right| \leqslant \frac{1}{2} .
\end{aligned}
$$

A conceptually straightforward derivation of the Bell inequalities for any case can now be imagined: Since $\boldsymbol{P}$ is local if $\boldsymbol{q}$ can be found which is non-negative, one may start with $0 \leqslant \boldsymbol{q}=\boldsymbol{R}^{\otimes 2 m} \boldsymbol{z}$ as inequalities for the correlations, i.e., the coefficients of $z$, and reduce the inequalities to find the constraints on only the observable correlations, those determined by $\boldsymbol{P}$ by, e.g., Fourier-Motzkin elimination. With enough stamina, one may hope to gain some analytical insight this way. Computationally, however, this quickly becomes intractable. Alternatively, one can proceed as usual, write down the deterministic strategies (the extremal points of the local polytope) but now in terms of the correlation basis, and use standard LP algorithms to obtain Bell inequalities. However, there is no efficiency gain over working in the space of $\boldsymbol{P}$ 's in this case and it also quickly becomes intractable to derive all Bell inequalities.

That leaves us with another approach, namely, to take a given $\boldsymbol{P}$ and try to determine which type of correlation it is. In Fig. 2, a schematic representation is given of the space of behaviors $\boldsymbol{P}$, showing the local, quantum, and nonsignaling sets. We will only distinguish between quantum and nonsignaling behaviors, but in case $\boldsymbol{P}$ is nonlocal we will also want to quantify the nonlocality. This problem is also considered in Ref. [13]. Here we will show how techniques from compressed sensing can be used to solve this problem efficiently.

We know that $\boldsymbol{P}$ is local if it corresponds to a joint probability distribution $\boldsymbol{q}$, i.e., if it solves the linear equation,

$$
\boldsymbol{P}=(\boldsymbol{D} \otimes \boldsymbol{D}) \boldsymbol{q}=(\boldsymbol{U} \boldsymbol{S} \otimes \boldsymbol{U} \boldsymbol{S}) \boldsymbol{z},
$$

with $q_{a_{1} \ldots a_{m} b_{1} \ldots b_{m}} \geqslant 0$. In the last section, we discussed that $z$, which can be regarded as $\boldsymbol{q}$ in a basis of singular vectors of $\boldsymbol{D} \otimes \boldsymbol{D}$, can be viewed as a generalized correlation representation.

It is not hard to find a solution to Eq. (32). We can use the explicit SVD of $\boldsymbol{D}$ Eqs. (15)-(17) to compute the MoorePenrose pseudoinverse [40] of $\boldsymbol{D} \otimes \boldsymbol{D}$ by inverting all nonzero singular values and apply it on $\boldsymbol{P}$ to get a quasiprobability $\boldsymbol{q}$. It corresponds to taking $\boldsymbol{z}$ with all coefficients not fixed by $\boldsymbol{P}$ (the nonobservable correlations) put to zero. This gives the solution of the linear equation Eq. (32) of minimal $\ell_{2}$ norm. While a viable quasiprobability is consistent with $\boldsymbol{P}$, there is no guarantee that this $\boldsymbol{q}$ is a probability if $\boldsymbol{P}$ is local. Adding any element $\boldsymbol{k}$ of the kernel of $\boldsymbol{D} \otimes \boldsymbol{D}$ to $\boldsymbol{q}$ gives a $\boldsymbol{q}^{\prime}=\boldsymbol{q}+\boldsymbol{k}$ that is still a quasiprobability and reproduces $\boldsymbol{P}$. It may happen that $\boldsymbol{q}$ with minimal $\ell_{2}$ norm lies outside the probability simplex but some $\boldsymbol{q}^{\prime}$ lies inside the probability simplex.

Let us consider as an example $n=m=2$ and $P(00 \mid x y)=$ $\delta_{a 0} \delta_{b 0}$. The correct hidden variable probability that generates this behavior is

$$
\boldsymbol{q}^{\prime}=\left[\begin{array}{llll}
1 & 0 & 0 & 0 \\
0 & 0 & 0 & 0 \\
0 & 0 & 0 & 0 \\
0 & 0 & 0 & 0
\end{array}\right],
$$

but the minimal $\ell_{2}$-norm solution is in fact

$$
\boldsymbol{q}=\frac{1}{16}\left[\begin{array}{cccc}
9 & 3 & 3 & -3 \\
3 & 1 & 1 & -1 \\
3 & 1 & 1 & -1 \\
-3 & -1 & -1 & 1
\end{array}\right]
$$

The situation is illustrated in Fig. 2(b). Clearly, $\ell_{2}$-norm minimization will in general not find a probability for $\boldsymbol{q}$ even when $\boldsymbol{P}$ is local.

To formulate the optimization problem that does implement a test for locality of $\boldsymbol{P}$, let us first introduce the concept of negativity. Let $\operatorname{neg}(\boldsymbol{q})=\sum_{\mathrm{a}_{1} \ldots \mathrm{b}_{\mathrm{m}}} \max \left(-\mathrm{q}_{\mathrm{a}_{1} \ldots \mathrm{b}_{\mathrm{m}}}, 0\right)$ be the negativity of $\boldsymbol{q}$ and define the negativity of $\boldsymbol{P}$ as the minimal neg $\boldsymbol{q}$ among all $\boldsymbol{q}$ compatible with $\boldsymbol{P}$. This appears as a natural quantifier of nonlocality in our setup. In the Appendix, we provide a proof that neg $(\boldsymbol{P})$ indeed satisfies basic properties of a valid measure of nonlocality. Clearly, neg $\boldsymbol{P}=0$ if and only if $\boldsymbol{P}$ is local.

In compressed sensing, one is interested in solving the linear equation $\boldsymbol{A} \boldsymbol{x}=\boldsymbol{b}$ and find the solution $\boldsymbol{x}$ that is as sparse as possible. Formally, the most sparse solution minimizes the $\ell_{0}$ norm. However, in practice one solves the equation while minimizing the $\ell_{1}$ norm which has the benefit of being convex and amenable to the techniques of convex optimization. This is known to yield surprisingly good results for signal recovery with many interesting applications. A discussion of these and 
of the underlying theory are beyond the scope of this paper and we refer the interested reader to the literature [20-23].

The relation of compressed sensing to our problem becomes clear once we notice that $\operatorname{neg} \boldsymbol{q}=\frac{1}{2}\|\boldsymbol{q}\|_{1}-1$. Hence, minimizing the negativity of all $\boldsymbol{q}$ compatible with $\boldsymbol{P}$ corresponds exactly to $\ell_{1}$-norm minimization in the following optimization problem:

$$
\begin{array}{ll}
\operatorname{minimize} & \|\boldsymbol{q}\|_{1} \\
\text { subject to } & (\boldsymbol{D} \otimes \boldsymbol{D}) \boldsymbol{q}=\boldsymbol{P} .
\end{array}
$$

This problem is known as basis pursuit in computer science literature. There are several classes of algorithms equipped to solve this. A well-known way is to map the problem to a linear program (LP). Although slightly differently formulated, this is very similar to the approach detailed in Ref. [13]. The problem with LPs is, however, that, while accurate, for large dimensions they become computationally expensive. The dimension of the hidden-variable space grows as $n^{2 m}$.

We will follow a different route here: Formulated in the correlation basis, our problem precisely fits the most efficient version of NESTA (shorthand for Nesterov's algorithm), a class of algorithms introduced in Ref. [35] to tackle exactly the basis pursuit problem.

Consider the minimization problem:

$$
\begin{array}{ll}
\operatorname{minimize} & \|\mathcal{V} z\|_{1} \\
\text { subject to } & \Pi z=z_{0} .
\end{array}
$$

Here $\mathcal{V}=\boldsymbol{V} \otimes \boldsymbol{V}=\boldsymbol{R}^{\otimes 2 m}$ is the basis transformation between the correlation basis and standard basis for the joint quasiprobability $\boldsymbol{q}$, the correlation vector $\boldsymbol{z}$ consists of two parts $z_{0}=$ $\Pi z$ of coefficients fixed by $\boldsymbol{P}$ (the observable correlations) and $z_{1}=(\mathbf{1}-\boldsymbol{\Pi}) z$ the coefficients that are not fixed by $\boldsymbol{P}$ (none observable correlations), which are the free parameters of the optimization. The tensor $\Pi$ is the projector onto the observable correlations, i.e., onto the subspace $\operatorname{ker}(\boldsymbol{S} \otimes \boldsymbol{S})^{\perp}$, and $\mathbf{1}-\boldsymbol{\Pi}$ projects onto $\operatorname{ker}(\boldsymbol{S} \otimes \boldsymbol{S})$. To obtain $\boldsymbol{z}_{0}$ from $\boldsymbol{P}$, it is useful to define the transformation tensor $\boldsymbol{T}=\left(\tilde{\boldsymbol{S}}^{-1} \boldsymbol{U}^{T} \otimes\right.$ $\tilde{\boldsymbol{S}}^{-1} \boldsymbol{U}^{T}$ ) where $\tilde{\boldsymbol{S}}^{-1}$ is obtained from the singular value tensor $S$ by taking the inverse of all the nonzero singular values, leaving the zeros untouched. Then we can compute $z_{0}=\boldsymbol{T P}$.

That brings us to our numerical results. We have used the NESTA package for Matlab [41] to solve the optimization problem Eq. (36). From the resulting $z$, we compute $\operatorname{neg}(\boldsymbol{P})=$ $\operatorname{neg}(\mathcal{V} z)$, which is the negativity of the optimal $\boldsymbol{q}=\mathcal{V} z$ of optimization problem Eq. (35) [42].

As reference, we have compared the NESTA results with the LP-based method from Ref. [13] implemented in Mathematica. The LP method minimizes the $\ell_{1}$ distance of $\boldsymbol{P}$ with the local set denoted NL $(\boldsymbol{P})$. To determine locality of $\boldsymbol{P}$, both methods have to set a threshold below which neg $(\boldsymbol{P})$ or $\mathrm{NL}(\boldsymbol{P})$ is taken to be zero due to finite precision. In both methods, the precession can be controlled by tuning certain parameters. In the case of NESTA, these parameters are muf, determining the smoothing of the $\ell_{1}$ norm [43], and TolVar which determines the convergence criterion. In case a high precision result is required, the output $z$ can easily be fed into a subsequent iteration with more stringent parameter settings.

To obtain test data, we sampled $100 \boldsymbol{P}$ for $n=2,3, \ldots, 20$ within the triangle $\boldsymbol{P}=c_{0} \boldsymbol{P}_{\mathrm{wn}}+c_{1} \boldsymbol{P}_{\mathrm{ld}}+\left(1-c_{0}-c_{1}\right) \boldsymbol{P}_{\mathrm{pr}}$
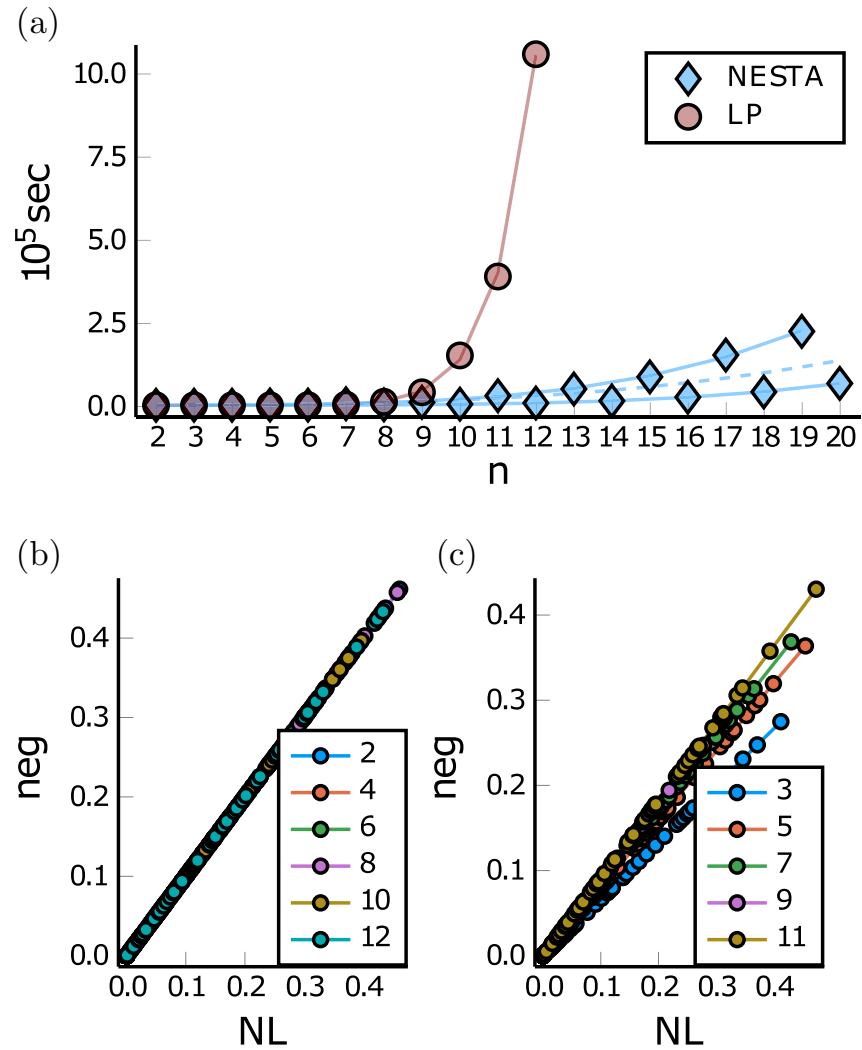

FIG. 3. Comparison of NESTA algorithm for determining neg $(\boldsymbol{P})$ and linear programming (LP) algorithm determining NL $(\boldsymbol{P})$. (a) To check efficiency, we computed computation times of NESTA and LP on 100 correlations $\boldsymbol{P}$ for the Bell $22 n$ scenario (bipartite, two inputs, $n$ outputs) for $n=2,3, \ldots, 20$ randomly sampled as described in the text. Solid lines are fits of the data with a power law $t=\lambda n^{\alpha}$. We chose this fit since both algorithms are expected to scale polynomially with the dimension of the problem which is $n^{4}$ for two inputs and two parties. Values for $\alpha$ and $\lambda$ can be found in Table I. We roughly find $\alpha \sim 4$ for NESTA while $\alpha \sim 11$ for LP. (b) To check accuracy of the NESTA method, we compared the computed neg $(\boldsymbol{P})$ with $\mathrm{NL}(\boldsymbol{P})$. We find that neg $(\boldsymbol{P}) \propto \mathrm{NL}(\boldsymbol{P})$ with exact equality when $n$ is even (left plot) but an $n$-dependent prefactor when $n$ is odd (right plot; values of $n$ are denoted in the legend).

with $0 \leqslant c_{0,1} \leqslant 1$ uniformly distributed random numbers. In other words, $\boldsymbol{P}$ is taken to be a convex combination of white noise $\boldsymbol{P}_{\mathrm{wn}}$ defined by $P_{\mathrm{wn}}(a b \mid x y)=1 / n^{2}$, a local deterministic point $\boldsymbol{P}_{\mathrm{ld}}$ (corner of the local set) defined by $P_{\mathrm{ld}}(a b \mid x y)=$ $\delta_{a 0} \delta_{b 0}$, and a generalized PR box $\boldsymbol{P}_{\mathrm{pr}}$ (nonlocal corner of the nonsignaling set) with $P_{\mathrm{pr}}(a b \mid x y)=1 / n$ if $b-a=x y$ mod 2. The sampled region corresponds schematically to the shaded triangle in Fig. 2(a).

We have compared the NESTA-based method with the LP-based method on accuracy and efficiency and present the results in Fig. 3. We ran both algorithms on the sampled data and stored the computation times and the values for the respective quantifiers of nonlocality. For NESTA, we used $\operatorname{muf}=$ TolVar $=10^{-10}$.

To test accuracy, we have compared the respective quantifiers $\operatorname{neg}(\boldsymbol{P})$ and $\mathrm{NL}(\boldsymbol{P})$ computed by the two algorithms, The comparison is plotted in Fig. 3(b). The two algorithms 
(a)

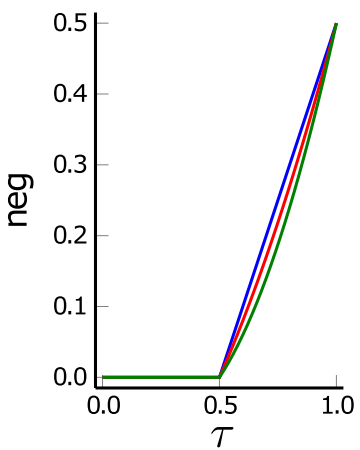

(b)

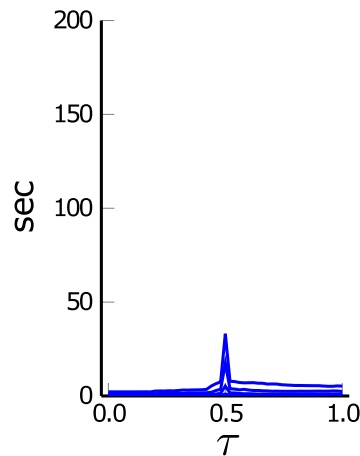

(c)

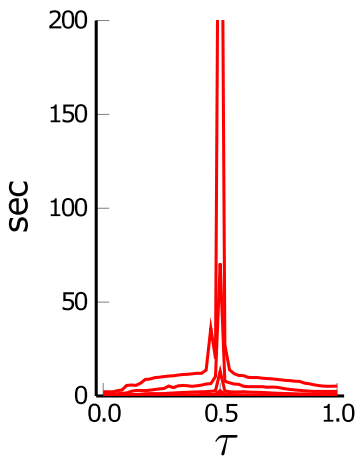

(d)

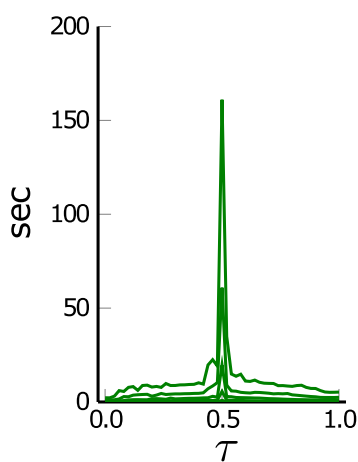

FIG. 4. Application of NESTA on a fixed path of behaviors $\boldsymbol{P}_{\tau}$ as described in the text for $n=2,4, \ldots, 12$. (a) The negativity neg $\left(\boldsymbol{P}_{\tau}\right)$ is independent of $n$ for even $n$. (b) The computation times to compute neg $\left(\boldsymbol{P}_{\tau}\right)$ using NESTA for the three paths from white noise to PR box. The computation times increase for increasing $n$, but the important observation is that the computation becomes much more costly when $\tau \sim 0.5$, which corresponds to the transition from local to nonlocal correlations. The three plots correspond to the choices of $\gamma=0$ (blue), $\gamma=0.5$ (red), and $\gamma=1.0$ (green), where $\gamma$ determines how the path takes the local deterministic corner $\boldsymbol{P}_{\mathrm{ld}}$ into account. Surprisingly, when $\gamma=0.5$, we find the highest peak and not when $\gamma=1$. The height of the peak is 436 seconds in that case.

agree in all cases in their respective determination whether $\boldsymbol{P}$ is local with a threshold of $10^{-10}$ (the number of local points in the case of our sampled data ranged from 36/100 for $n=20$ to $61 / 100$ for $n=9$ ). These points all collapse on the origin of Fig. 3(b). For the nonlocal points, we have compared the respective quantifiers of nonlocality and find that they are proportional for all $n$, but curiously, for even $n$, we find exact equality $\operatorname{neg}(\boldsymbol{P})=\mathrm{NL}(\boldsymbol{P})$ while for odd $n$, we find a nontrivial, $n$-dependent constant of proportionality neg $(\boldsymbol{P})=$ $\beta_{\mathrm{n}} \mathrm{NL}(\boldsymbol{P})$. An explanation of the difference between even and odd $n$ is left for future research.

Up to the aforementioned questions of precision, we thus find that the NESTA-based method serves as a perfect test of nonlocality, similar to the LP method. The perfect accuracy can be understood from the convexity of the optimization problem which guarantees convergence to the global minimum. We note that, for local correlations, the minimum may be degenerate as it corresponds to the intersection of the affine subspace of solutions of Eq. (32) and the probability simplex on which the negativity is equal to zero. An interesting question is whether the method can be generalized to other causal scenarios, but a possible obstacle may be that the corresponding optimization turns out not to be convex.

To test efficiency of the NESTA-based method, we compared the computation time of processing the hundred sampled points with the computation time for the LP method for each $n$. The results are plotted in Fig. 3(a). For both methods, we have only recorded the time of the analysis of the data, neglecting the setup calculations that need to be done only once for a fixed $n$ and hence do not scale with the number of data points. We observed that, from about $n=12$, the LP method became impractically slow, taking more than a day to complete the data set, so we aborted the calculation. The NESTA-based method had no trouble finishing all the sampled data well within a day. Both computations ran on the same desktop computer. In the plot in Fig. 3(a), we clearly see that NESTA has much better scaling when $n$ grows large. Although by no means a perfect benchmark of the actual efficiency of the two algorithms, we take it as an indication that in these scenarios NESTA offers a significant efficiency benefit when $n$ becomes large. For the NESTA algorithm, we find a curious difference in even and odd $n$ cases, where even $n$ is more efficient. It appears NESTA needs on average more iterations in the odd $n$ case. Fitting the time with a power law $\propto n^{\alpha}$, we find exponents $\alpha$ between 3 and 4 for NESTA while $\alpha \sim 11$ for LP (see Table I).

To get more insight into the NESTA computation, we also ran the code for even $n=2,4, \ldots, 12$ on three fixed paths $\boldsymbol{P}_{\tau}$ we have chosen to run from the white noise correlation to the generalized PR box as (1) a straight line, (2) a kinked line through the middle of the sampled triangle, and (3) a path traversing the edge of the sampled triangle passing by the local deterministic point [see Fig. 2(a) for an illustration]. Explicitly, we have defined $\boldsymbol{P}_{\tau}=\left(1-\gamma c_{\tau}\right)\left[(1-\tau) \boldsymbol{P}_{\mathrm{wn}}+\right.$ $\left.\tau \boldsymbol{P}_{\mathrm{pr}}\right]+\gamma c_{\tau} \boldsymbol{P}_{\mathrm{ld}}$, where $c_{\tau}=1-2|\tau-0.5|$ and have taken $\gamma=0.0,0.5,1.0$. We then plotted the negativity, which is independent of $n$ for even $n$, as well as the computation time as a function of $\tau$ (see Fig. 4). The value $\tau=0.5$ marks the transition from local to nonlocal correlations. It is at this value that we find a sharp peak in the computation time. As could be expected, the NESTA algorithm needs more iterations to reach convergence when the correlation is close to the boundary of the local set. It makes sense since, in terms of the joint quasiprobability $\boldsymbol{q}$, we are looking for a solution close to the edge of the probability simplex where the $\ell_{1}$ norm shows singular behavior. Somewhat surprisingly, it is not the local

TABLE I. Power-law fit of computation times in Fig. 3(a) as time $=\lambda n^{\alpha}$.

\begin{tabular}{lcc}
\hline \hline & $\lambda$ & $\alpha$ \\
\hline LP & $8.8 \cdot 10^{-8}$ & 11 \\
NESTA & 1.4 & 3.1 \\
NESTA even & 0.22 & 3.9 \\
NESTA odd & $1.1 \cdot 10^{-3}$ & 4.5 \\
\hline \hline
\end{tabular}


deterministic point that takes the most time but rather the intermediate case $\gamma=0.5$.

One may wonder what the use of tensor networks is at this point. Indeed, the NESTA algorithm takes matrix and vector inputs and really just uses linear algebra. While there certainly is a convenience benefit in using tensors-we found it very useful to construct the input data for NESTA using the NCON function [44] for tensor network manipulations in Matlab - the performance benefit may not be so clear at this point. But a quick analysis of the computational cost reveals that in fact there is a true gain in efficiency from using the particular tensor-network decomposition.

Each iteration of NESTA, when the problem is presented in the form Eq. (35), has the computational cost of about two matrix multiplications, namely, with the matrices $\mathcal{V}$ and $\mathcal{V}^{T}$, of dimension $n^{2 m}=n^{4}$ [35]. In our application, the matrix $\mathcal{V}=\boldsymbol{R}^{\otimes 2 m}$ is in fact a tensor product operator. This means the multiplication can be implemented more efficiently without constructing the full matrix. We leveraged this by using the kronmult function [45] in our algorithm. We thus see that the efficiency gain we found is indeed an interplay of the theory of compressed sensing with the tensor-network decomposition of $\boldsymbol{P}$ and $\boldsymbol{D}$.

From the above, we conclude that the sparse recovery method NESTA from the theory of compressed sensing can be used to find the quasiprobability $\boldsymbol{q}$ for the hidden variable that has minimal $\ell_{1}$ norm leads to the observable behavior $\boldsymbol{P}$ accurately and efficiently. Since $\ell_{1}$-norm minimization is equivalent to minimizing the negativity of $\boldsymbol{q}$, we are thus guaranteed that if $\boldsymbol{P}$ is local we find a true probability for $\boldsymbol{q}$. In practice, we use the correlation basis which fits the most efficient formulation of NESTA.

\section{CONCLUSIONS}

Bell nonlocality is a cornerstone of quantum theory and central resource in a variety of quantum information processing protocols. For that aim, a basic step is to decide whether a given observed correlation is nonlocal or not. Given its importance, over the years a few approaches have been developed to tackle the problem [2,13,46-50], however, most are limited in practice to relatively simple Bell scenarios, with a small number of distant parties or measurement settings and outcomes. Most such approaches are based on a geometric view of Bell scenarios, more precisely, the fact that local correlations form a polytope and that to detect nonlocality means to find ways of certifying that a given correlation lies outside this local set. However, different perspectives such as sheaf theoretic [14], graph theoretical [15], causal [17,18], or category theoretic [51-53] are also possible. Often a different perspective leads to additional insights and computational methods.

Here we propose a perspective to understand and quantify Bell nonlocality, based on a tensor network description. With that, we showed that nonsignaling correlations can be described by hidden variable models governed by quasiprobabilities, the negativity of which offers a natural way to quantify nonlocality. By refining our description via a SVD, we obtained a natural basis to attack the problem of quantifying nonlocality and that can be computationally implemented via extremely efficient sparse recovery algorithms. To show its relevance, we compared the NESTA algorithm used in compressed sensing with the standard LP tool used in the study of nonlocality and showed a significant speedup in computational time as a function of the complexity of the Bell scenario.

We believe this perspective on Bell's theorem opens a few venues for future research. Local correlations are equivalent to hidden variable models governed by probabilities while general nonsignaling correlations imply quasiprobabilities. What are the restrictions imposed by Born's rule (the quantum mechanical rule) to such quasiprobabilities? There is an important research program trying to recover the quantum limitations on correlations [36,54], however, to our knowledge, the intersection of this program with this quasiprobability description of nonlocality has not been considered so far (see, however, recent results on general connections between quantum theory and quasiprobabilities such as Refs. $[27,34,55])$. A potential application of the sparse recovery method is to combine it with the machine-learning approach that has been recently proposed [50]. There, neural networks are used in supervised learning algorithms that create a machine model of the local set. The bottleneck of the method is exactly the fact that the standard LP approach is used to generate the input data, consisting of a set of correlations and their respective degree of nonlocality. Can the NESTA algorithm [35] provide a more scalable solution to this machine learning approach? Finally, the tensor network description can easily be extended to more complex Bell scenarios consisting of several independent sources [56] and leading to nonconvex sets of correlations $[57,58]$. Can generalizations of sparse recovery algorithms adapted to deal with nonlinear constraints [59] provide a way to deal with such complicated Bell scenarios? Can tensor network ideas be further leveraged to manage computational costs if the network of causal relations increases in size in a certain way? We hope our results motivate further research along these directions.

\section{ACKNOWLEDGMENTS}

We acknowledge the John Templeton Foundation via Grant Q-CAUSAL No. 61084, the Serrapilheira Institute (Grant No. Serra-1708-15763), the Brazilian National Council for Scientific and Technological Development (CNPq) via the National Institute for Science and Technology on Quantum Information (INCT-IQ), and Grants No. 307172/2017-1 and No. 406574/2018-9, the Brazilian agencies MCTIC and MEC.

\section{APPENDIX}

Here, our objective is to prove that $\operatorname{neg}(\boldsymbol{P})$ is a valid quantifier of nonlocality, explicitly showing its invariance under local relabelings and monotonicity under convex combination with local distributions and postprocessing. The quantifier $\operatorname{neg}(\boldsymbol{P})$ is the minimal negativity $\operatorname{neg}(\boldsymbol{q})=$

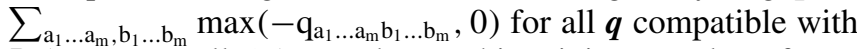
$\boldsymbol{P}$ (a set we call $C_{\boldsymbol{P}}$ ). We denote this minimum value of $\boldsymbol{q}$ as $\boldsymbol{q}^{*}$. We also have that $\operatorname{neg}(\boldsymbol{q})=\frac{1}{2}\|\boldsymbol{q}\|_{1}-1$. 
Proof. First we show that neg $(\boldsymbol{P})$ is invariant under relabelings of the inputs and outputs, an operation we denote by $\mathcal{R}$. We have that

$$
\begin{aligned}
\operatorname{neg}(\mathcal{R}(\boldsymbol{P})) & =\min _{\boldsymbol{q} \in C_{\boldsymbol{P}}} \sum_{a_{1} \ldots a_{m}, b_{1} \ldots b_{m}} \max \left(\mathcal{R}\left(-q_{a_{1} \ldots a_{m} b_{1} \ldots b_{m}}\right), 0\right) \\
& =\min _{\boldsymbol{q} \in C_{\boldsymbol{P}}} \sum_{a_{1} \ldots a_{m}, b_{1} \ldots b_{m}} \max \left(-q_{a_{1} \ldots a_{m} b_{1} \ldots b_{m}}, 0\right) \\
& =\operatorname{neg}(\boldsymbol{P})
\end{aligned}
$$

where the equality follows from the fact that we are summing over all values and $\mathcal{R}\left(-q_{a_{1} \ldots a_{m} b_{1} \ldots b_{m}}\right)$ is obtained from $q_{a_{1} \ldots a_{m} b_{1} \ldots b_{m}}$ simply by a permutation of entries (all entries being covered by the sum).

Second, consider that we make a convex combination of $\boldsymbol{P}$ with a vector $\boldsymbol{P}_{L}$ that is local, that is, we obtain distribution $\pi \boldsymbol{P}+(1-\pi) \boldsymbol{P}_{L}$, where $\pi \in[0,1]$. Since $\boldsymbol{P}$ is local we have a joint (positive) probability distribution $q_{a_{1} \ldots a_{m} b_{1} \ldots b_{m}}^{L}$ describing it. As we prove next, it follows that $\operatorname{neg}\left(\pi \boldsymbol{P}+(1-\pi) \boldsymbol{P}_{\mathrm{L}}\right) \leqslant$ $\operatorname{neg}(\boldsymbol{P})$, thus proving monotonicity under convex combinations. We have that

$$
\begin{aligned}
\operatorname{neg}\left(\pi \boldsymbol{P}+(1-\pi) \boldsymbol{P}_{L}\right) & =\min _{\substack { \boldsymbol{q} \in C_{\boldsymbol{P}} \\
\begin{subarray}{c}{a_{1} \ldots a_{m} \\
b_{1} \ldots b_{m}{ \boldsymbol { q } \in C _ { \boldsymbol { P } } \\
\begin{subarray} { c } { a _ { 1 } \ldots a _ { m } \\
b _ { 1 } \ldots b _ { m } } }\end{subarray}} \max \left(-\pi q_{a_{1} \ldots a_{m} b_{1} \ldots b_{m}}-(1-\pi) q_{a_{1} \ldots a_{m} b_{1} \ldots b_{m}}^{L}, 0\right) \\
\leqslant & \min _{\substack { \boldsymbol{q} \in C_{\boldsymbol{P}} \\
\begin{subarray}{c}{a_{1} \ldots a_{m} \\
b_{1} \ldots b_{m}{ \boldsymbol { q } \in C _ { \boldsymbol { P } } \\
\begin{subarray} { c } { a _ { 1 } \ldots a _ { m } \\
b _ { 1 } \ldots b _ { m } } }\end{subarray}} \max \left(-q_{a_{1} \ldots a_{m} b_{1} \ldots b_{m}}, 0\right) \\
& =\pi \operatorname{meg}(\boldsymbol{P})
\end{aligned}
$$

Finally, we prove the monotonicity of $\operatorname{neg}(\boldsymbol{P})$ under post-processing operations $\mathcal{O}$, that is, $\operatorname{neg}(\mathcal{O}(\boldsymbol{P})) \leqslant \operatorname{neg}(\boldsymbol{P})$. Given $P$, a post-processing operation transforms $\boldsymbol{P}$ into $\mathcal{O}(\boldsymbol{P})$, where

$$
\mathcal{O}(\boldsymbol{P})(\alpha, \beta \mid x, y)=\sum_{a, b} O^{L}(\alpha, \beta \mid a, b, x, y) \times P(a, b \mid x, y),
$$

and $O^{L}$ is a probability distribution with inputs $a, b$ and outputs $\alpha, \beta$ satisfying

$$
O^{L}(\alpha, \beta \mid a, b, x, y)=\sum_{\lambda} p(\lambda) O_{A}^{L}(\alpha \mid a, x) \times O_{B}^{L}(\beta \mid b, y) .
$$

We have that

$$
\begin{aligned}
& \operatorname{neg}(\mathcal{O}(\boldsymbol{P}))=\min _{\boldsymbol{q} \in C_{\boldsymbol{P}}} \frac{1}{2}\|\mathcal{O}(\boldsymbol{q})\|_{1}-1 \\
& \leqslant \frac{1}{2}\left\|\mathcal{O}\left(\boldsymbol{q}^{*}\right)\right\|_{1}-1 \\
& =\frac{1}{2} \sum_{\substack{\alpha_{1}, \ldots, \alpha_{m} \\
\beta_{1}, \ldots, \beta_{m}}}\left|\mathcal{O}\left(\boldsymbol{q}^{*}\right)\right|-1 \\
& =\frac{1}{2} \sum_{\substack{\alpha_{1}, \ldots, \alpha_{m} \\
\beta_{1}, \ldots, \beta_{m}}}\left|\sum_{\substack{a_{1}, \ldots, a_{m} \\
b_{1}, \ldots, b_{m}}} O^{L}\left(\alpha_{1}, \ldots, \alpha_{m}, \beta_{1}, \ldots, \beta_{m} \mid a_{1}, \ldots, a_{m}, b, \ldots, b_{m}\right) q_{a_{1}, \ldots, a_{m}, b, \ldots, b_{m}}^{*}\right|-1 \\
& \leqslant \frac{1}{2} \sum_{\begin{array}{c}
\alpha_{1}, \ldots, \alpha_{m} \\
\beta_{1}, \ldots, \beta_{m} \\
a_{1}, \ldots, a_{m} \\
b_{1}, \ldots, b_{m}
\end{array}} O^{L}\left(\alpha_{1}, \ldots, \alpha_{m}, \beta_{1}, \ldots, \beta_{m} \mid a_{1}, \ldots, a_{m}, b_{1}, \ldots, b_{m}\right)\left|q_{a_{1}, \ldots, a_{m}, b, \ldots, b_{m}}^{*}\right|-1 \\
& =\frac{1}{2} \sum_{\substack{a_{1}, \ldots, a_{m} \\
b_{1}, \ldots, b_{m}}}\left|q_{a_{1}, \ldots, a_{m}, b_{1}, \ldots, b_{m}}^{*}\right|-1 \\
& =\frac{1}{2}\left\|\boldsymbol{q}^{*}\right\|_{1}-1 \\
& =\operatorname{neg}(\boldsymbol{P}) \text {. }
\end{aligned}
$$


[1] J. S. Bell, Physics 1, 195 (1964).

[2] N. Brunner, D. Cavalcanti, S. Pironio, V. Scarani, and S. Wehner, Rev. Mod. Phys. 86, 419 (2014).

[3] S. Pironio, A. Acín, S. Massar, A. B. de La Giroday, D. N. Matsukevich, P. Maunz, S. Olmschenk, D. Hayes, L. Luo, T. A. Manning et al., Nature 464, 1021 (2010).

[4] A. K. Ekert, Phys. Rev. Lett. 67, 661 (1991).

[5] H. Buhrman, R. Cleve, S. Massar, and R. de Wolf, Rev. Mod. Phys. 82, 665 (2010).

[6] D. Mayers and A. Yao, Quantum Inf. Comput. 4, 273 (2004).

[7] A. Acín, N. Brunner, N. Gisin, S. Massar, S. Pironio, and V. Scarani, Phys. Rev. Lett. 98, 230501 (2007).

[8] J. I. De Vicente, J. Phys. A: Math. Theor. 47, 424017 (2014).

[9] I. Pitowsky, Quantum Probability-Quantum Logic (SpringerVerlag, Berlin, 1989).

[10] J. F. Clauser, M. A. Horne, A. Shimony, and R. A. Holt, Phys. Rev. Lett. 23, 880 (1969).

[11] D. Collins, N. Gisin, N. Linden, S. Massar, and S. Popescu, Phys. Rev. Lett. 88, 040404 (2002).

[12] D. Collins and N. Gisin, J. Phys. A: Math. Gen. 37, 1775 (2004).

[13] S. G. A. Brito, B. Amaral, and R. Chaves, Phys. Rev. A 97, 022111 (2018).

[14] S. Abramsky and A. Brandenburger, New J. Phys. 13, 113036 (2011).

[15] A. Acín, T. Fritz, A. Leverrier, and A. B. Sainz, Commun. Math. Phys. 334, 533 (2015).

[16] T. Fritz, New J. Phys. 14, 103001 (2012).

[17] C. J. Wood and R. W. Spekkens, New J. Phys. 17, 033002 (2015).

[18] R. Chaves, R. Kueng, J. B. Brask, and D. Gross, Phys. Rev. Lett. 114, 140403 (2015)

[19] R. Orús, Nat. Rev. Phys. 1, 538 (2019).

[20] D. L. Donoho, IEEE Trans. Inf. Theory 52, 1289 (2006).

[21] E. J. Candès, in Proceedings of the International Congress of Mathematicians Madrid, August 22-30, 2006 (European Mathematical Society, 2007), pp. 1433-1452.

[22] Y. C. Eldar and G. Kutyniok, Compressed Sensing: Theory and Applications (Cambridge University Press, Cambridge, 2012).

[23] A. Stern, Optical Compressive Imaging (CRC Press, Boca Raton, FL, 2016).

[24] B. Coecke and A. Kissinger, Picturing Quantum Processes (Cambridge University Press, Cambridge, 2017).

[25] B. Coecke and A. Kissinger, Int. J. Quantum. Inform. 14, 1640020 (2016).

[26] J. D. Biamonte, S. R. Clark, and D. Jaksch, AIP Adv. 1, 042172 (2011).

[27] J. v. d. Wetering, Electron. Proc. Theor. Comp. Sci. 266, 179 (2018).

[28] B. Fong, arXiv:1301.6201.

[29] J. C. Baez and T. Fritz, Theory Appl. Categories 29, 422 (2014).

[30] S. R. White, Phys. Rev. Lett. 69, 2863 (1992).

[31] U. Schollwöck, Ann. Phys 326, 96 (2011).

[32] M. A. Nielsen, Neural Networks and Deep Learning (Determination Press, San Francisco, CA, 2015), Vol. 2018.

[33] S. Abramsky and A. Brandenburger, in Horizons of the Mind. A Tribute to Prakash Panangaden (Springer, Cham, 2014), pp. 59-75.
[34] M. Appleby, C. A. Fuchs, B. C. Stacey, and H. Zhu, Eur. Phys. J. D 71, 197 (2017).

[35] S. Becker, J. Bobin, and E. Candès, SIAM J. Imaging Sci. 4, 1 (2011).

[36] S. Popescu and D. Rohrlich, Found. Phys. 24, 379 (1994).

[37] A. Fine, Phys. Rev. Lett. 48, 291 (1982).

[38] The reader familiar with tensors from the theory of general relativity (GR) may be confused that there are no requirements related to a change of variables. In GR the word tensor actually refers to a tensor field and a change of coordinates on the base manifold transforms both the coefficients and the basis. The requirement of the transformation of tensor coefficients ensures consistency with the basis transformation such that they define a geometric object independent of choice of coordinates. In our case, no such requirement exists and any multi-dimensional array defines a tensor with respect to some fixed basis.

[39] R. W. Spekkens, Phys. Rev. A 71, 052108 (2005).

[40] R. Penrose, in Mathematical Proceedings of the Cambridge Philosophical Society (Cambridge University Press, Cambridge, 1955), Vol. 51, pp. 406-413.

[41] NESTA: A Fast and Accurate First-order Method for Sparse Recovery, https://statweb.stanford.edu/ candes/software/nesta (2009).

[42] Code for this project can be found at https://github.com/ SebasEliens/N2-bell-recovery.

[43] The Huber function $f_{\mu}$ is used to replace the $\ell_{1}$ norm. They are equal for large arguments but $f_{\mu}(x)=x^{2} /(2 \mu)$ for $\|x\|<\mu$.

[44] R. N. C. Pfeifer, G. Evenbly, S. Singh, and G. Vidal, arXiv:1402.0939 [physics.comp-ph].

[45] D. Gleich, Fast and efficient kronecker multiplication, https:// www.mathworks.com/matlabcentral/fileexchange/23606-fastand-efficient-kronecker-multiplication (2020).

[46] E. A. Fonseca and F. Parisio, Phys. Rev. A 92, 030101(R) (2015).

[47] A. Montina and S. Wolf, arXiv:1609.06269.

[48] F. Baccari, D. Cavalcanti, P. Wittek, and A. Acín, Phys. Rev. X 7, 021042 (2017).

[49] C. Duarte, S. Brito, B. Amaral, and R. Chaves, Phys. Rev. A 98, 062114 (2018).

[50] A. Canabarro, S. Brito, and R. Chaves, Phys. Rev. Lett. 122, 200401 (2019).

[51] E. Wolfe, D. Schmid, A. B. Sainz, R. Kunjwal, and R. W. Spekkens, arXiv:1903.06311.

[52] D. Rosset, D. Schmid, and F. Buscemi, arXiv:1911.12462.

[53] D. Schmid, D. Rosset, and F. Buscemi, Quantum 4, 262 (2020).

[54] M. Navascués, S. Pironio, and A. Acín, Phys. Rev. Lett. 98, 010401 (2007).

[55] H. Zhu, Phys. Rev. Lett. 117, 120404 (2016).

[56] C. Branciard, N. Gisin, and S. Pironio, Phys. Rev. Lett. 104, 170401 (2010).

[57] R. Chaves, Phys. Rev. Lett. 116, 010402 (2016).

[58] D. Rosset, C. Branciard, T. J. Barnea, G. Pütz, N. Brunner, and N. Gisin, Phys. Rev. Lett. 116, 010403 (2016).

[59] H. Ohlsson, A. Y. Yang, R. Dong, M. Verhaegen, and S. S. Sastry, in Regularization, Optimization, Kernels, and Support Vector Machines (CRC Press, 2014), Chap. 9. 